\title{
On-the-job training of clinical officers in Malawi
}

\section{Peter Jiskoot}

Surgeon and Project Manager Clinical Officer Training in Malawi

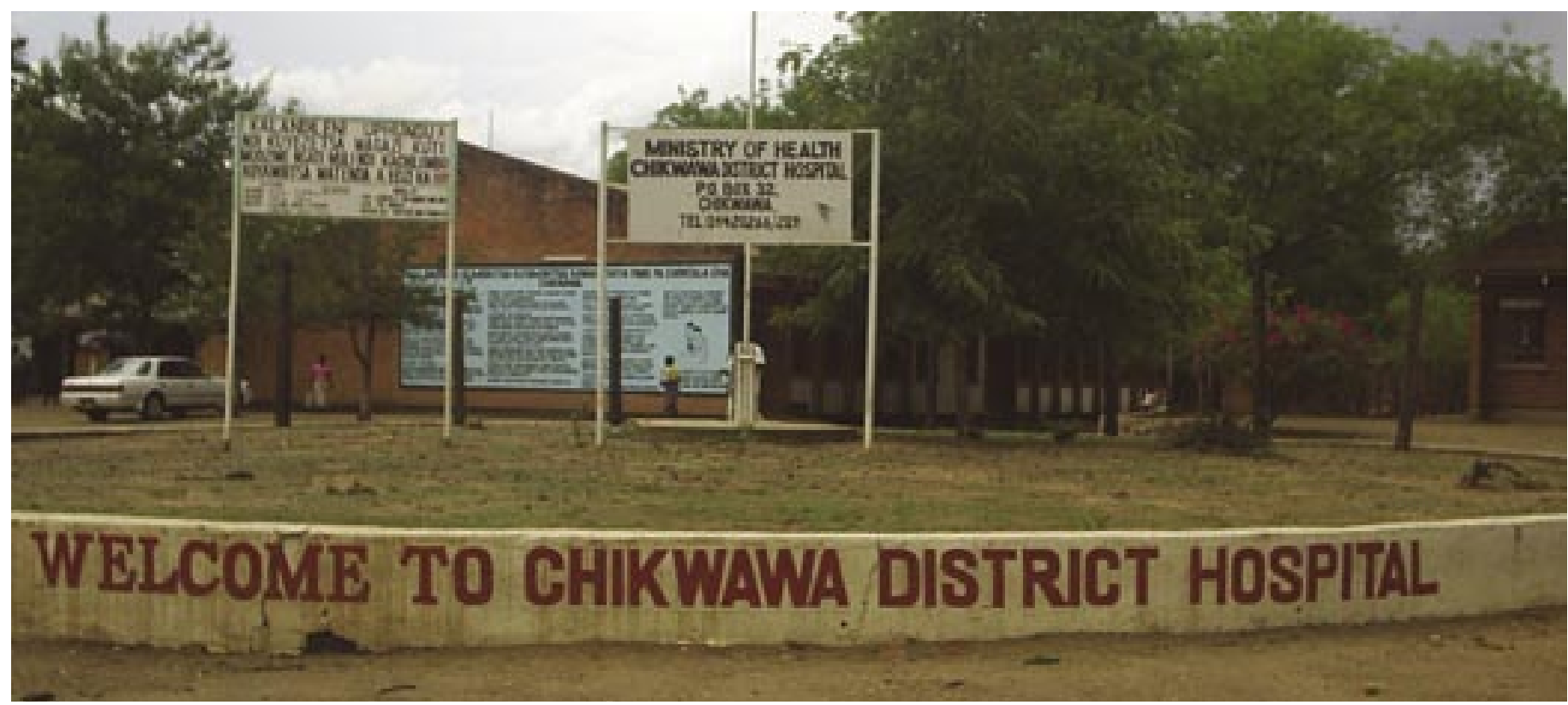

\section{The problem}

Although Malawi started a Medical College in 1991 to train medical doctors, it continues to face a chronic shortage of medical staff.

A number of qualified doctors and nurses continue to leave the country for better conditions abroad and there are very few specialist services available in District- and Missionhospitals. Currently there are 2 doctors and 6 nurses per 100,000 population ${ }^{1}$. The minimum numbers for developed countries are respectively 27 doctors and 100 nurses. Meanwhile Malawi has a huge disease burden (greatly augmented by HIV / Aids) and an unacceptably high mortality rate among both children and young adults. The maternal death rate in Malawi has been reported at 1800/100,000 in $2004^{2}$ and although this figure is disputed, Malawi's rate is almost certainly among the highest in the world. The average life expectancy has come down to 37 years for males, from 65 some 25 years ago ${ }^{3}$.

Owing to the shortage of doctors, clinical officers (COs) are the backbone of health care in Malawi. Most of the peripheral hospitals are managed by COs and this situation is unlikely to change for the coming years. COs are paramedicals (similar to nurse practitioners in some western countries) who are trained for four years at one of three existing institutions that offer such instruction in Malawi. After completion of the training they face a difficult job: they must work as medical officers in the hospitals but they lack sufficient knowledge and skills.

Most district hospitals have only one doctor, the district health officer (DHO), who is recruited straight from internship and is busy running the hospital and district as well as overseeing the clinical work. He or she often lacks many of the basic surgical skills.

Many patients presenting to district and mission hospitals require surgical treatment for trauma, obstetric, abdominal or orthopaedic emergencies. Owing to lack of surgical training and experience of COs, patients often have to be referred to one of the four existing central hospitals in the country. Those four central hospitals are already overloaded because for a considerable part they function as district hospitals for the surrounding area. A study published in 2004 reported that only $3 \%$ of the procedures done in the theatres of all 21 district hospitals in Malawi were 'general surgery', a fact that the authors attributed to the lack of knowledge and experience of $\mathrm{COs}^{4}$. Most procedures done in theatre by $\mathrm{COs}$ are obstetric and/or gynaecological. The lack of surgical experience in the hospitals may have contributed to the high frequency of complications and the high mortality that were also reported.

\section{A solution}

One sustainable solution is to upgrade the surgical and gynaecological knowledge and skills of clinical officers in district and mission-hospitals. The patient can then stay in his own area, does not have to travel long distances, can be treated faster and does not sustain extra costs from his already very limited financial resources. Moreover the central hospitals then can focus on the treatment of more complicated diseases and on the teaching of medical students.

To avoid reducing human resources at the district level even further by centralised training, we requested permission to train COs in their own hospitals, and to upgrade their surgical knowledge and skills by training on site and on the job.

\section{The start of the clinical officer project}

To address the problem, the Surgical Department of the College of Medicine in Blantyre supported the start of a project called: On the job training of surgical and gynaecological procedures in 8 District and 9 Mission Hospitals in the Southern region of Malawi.

The Project was carried out by three semi-retired Dutch surgeons working on rotation in 11 hospitals, and backup 
support was given at six of these hospitals by two German and two Dutch surgeons working at the Department of Surgery of the College of Medicine.

The Project owner is the Christian Health Association of Malawi (CHAM). Stakeholders of the project are the Ministry of Health, the Department of Surgery in the College of Medicine, and the three Malawi institutions for the training of Clinical Officers.

The project was financially supported by ICCO (Interchurch Organisation for Development Co-operation), a NGO in the Netherlands, and by private funding.

\section{Objectives of the Project}

1. To support advise and empower the medical teams of district hospitals, thus contributing to Continuing Medical Education (CME)

2. To specifically train selected COs in a selection of basic surgical procedures.

3. To focus on the treatment of common surgical problems and on the initial treatment for trauma, obstetric, abdominal and orthopedic emergencies

\section{Specific objectives}

1) To train COs to be competent in:

- Safe practice for common surgical and obstetrical procedures

- The care of trauma cases, including damage control surgery

- Stabilise the seriously injured patient before transfer to the Central Hospitals

2) In those hospitals with adequate facilities and where the COs have demonstrated improved surgical abilities, to encourage them to learn more complicated procedures.

3) To provide surgical and other textbooks such as: Primary surgery (ed. M.King), Surgical Care at the District Hospital (WHO), The Burns Manual and Common surgical problems in Malawi, (both edited by E. van Hasselt), The surgical protocols from the Department of surgery (DOS), Common skin diseases in Africa (ed Van Hees \& Naafs)

Entry requirements for a $\mathrm{CO}$ wishing to join the training programme

1. Registered $\mathrm{CO}$ who graduated from one of the training institutions in Malawi.

2. Has worked in hospital/s for at least 3 years

3. Has an interest in surgery

A maximum of 2 COs could be trained per hospital, and an initial total number of 25 COs were incorporated in the project.

\section{Activities from January 2005 - January 2007: Hospital day visits and attachment weeks}

The 2 selected clinical officers per hospital were visited in their own hospital by surgeons every 4 weeks.

A number of attachment weeks were centrally organized for extra theory and skills training in general surgery, anaesthesia, obstetrics and gynaecology, orthopaedics and trauma, ophthalmology and pathology. Examples are skills in Acute Trauma Life Support, bowel suturing, skin grafting and fine needle aspiration. Dermatology and ENT will soon be added to the programme.

Programme of a hospital day visit by the supervising surgeon

1. Select surgical patients who may require theatre

2. Make ward rounds and see all surgical and orthopaedic patients

3. Theatre: operations preferably to be done by the CO in training, assisted by the visiting surgeon

4. See selected patients at OPD, together with the trainee

5. Teach surgical topics - not only to the COs but also for all interested medical staff members, as part of continuing medical education.

6. Meet with the $\mathrm{DHO}$ or the Medical Director about the outcome of the day's visit. Advise how to improve the standard of surgical care in the hospital in general, and in the theatre in particular. Suggest the setting up of a theatre committee and a trauma team.

\section{Aim}

After 2 years the clinical officer should be able to perform the most common surgical procedures (inguinal hernia, hydrocele), but $\mathrm{s} /$ he should also be able to do damage control surgery (such as a colostomy for bowel obstruction, and bowel resection). The very best COs working in a good hospital are then trained to do more complicated operations like prostatectomy, hysterectomy and laparotomy.

\section{Number of COs}

The CO training programme started in January 2005 with 26 COs in training, working in 17 hospitals. In January 2006 the project continued the training with 23 COs working in 15 hospitals. Three COs dropped out because of serious illness. From June-August 2006, another 6 COs dropped out of the programme because they left their district hospitals. (Although the Ministry of Health $[\mathrm{MoH}]$ was requested not to transfer COs who had entered the two years' training, 4 left for an educational MoH course at the Mzuzu University and 2 took a job elsewhere).

Although almost all of the COs would have liked to continue and finish the training, the main reason for stepping out was that no career perspective had as yet been offered by the $\mathrm{MoH}$ to the $\mathrm{CO}$ at the end of the 2 years' training.

In January 2007, at the end of the 2 years training, 15 COs working in 12 hospitals received a certificate of attendance.

\section{Dutch medical student program}

All medical Universities in the Netherlands have been informed about the possibility for a maximum of 2 Dutch medical students to join the 'on-the-job' visits to the hospitals in a tour of 4 weeks each. In the last 3 years about 50 Dutch medical students have visited the Malawi hospitals as part of an elective period in surgery and/or gynaecology 


\section{International attention}

The on-the-job training has attracted the attention of international organizations as this style of training is regarded as less damaging to human resources than traditional courses that take trainees away from their place of work.

The World Health Organization visited the project in 2005. In recent years WHO has shown an increasing interest in district hospital surgery in Africa with the publication of the new WHO book "Surgery at the District Hospital". This book encourages surgery to be done as far as possible at a district level, instead of transferring all patients to tertiary centres.

\section{Assessment of the training}

Before the CO training started, we informed the Ministry of Health that we were organizing a training for the COs and not a course, and that no formal assessment by exams on the knowledge or skills would be performed.

Therefore evaluation was based on the COs' own feedback, the number of operations recorded in the theatre book, and the assessments by a questionnaire at the end of each attachment week. These assessments were combined with the supervising surgeon's observations.

A study on the outcome of the training was performed by Dr N.A. Phiri as part of a Masters in Public Health (MPH) degree at the College of Medicine ${ }^{6}$. He wrote " This training after one year definitely increased the number of bernia operations at the district level, not only for programme clinical officers but also for the other COs. Not only were the numbers increased but also the quality of the operation has improved in terms of non-wastage of precious antibiotics and post operation outcomes in terms of wound infection and wound separation."

The referrals to Queen Elizabeth Central Hospital have decreased but the numbers were not statistically significant.

\section{Recent developments}

We have offered our Powerpoint presentations on the surgical/gynaecological topics to the 3 existing teaching institutions for training COs in Malawi (Malawi Colleges of Health Sciences). We have also presented these three Colleges with surgical textbooks to be used by teachers and $\mathrm{CO}$ students. In this way we hope to contribute to the provision of uniform surgical protocols in all hospitals in Malawi. To standardize the (surgical) treatment of patients is important, as Malawi is visited by an increasing number of specialists from all over the world and they all bring in their own views of treating patients. This often creates a confusion that could be avoided.

All surgical departments of the 4 existing central hospitals in Malawi are involved in one way or another in visiting district hospitals and in seeing patients and doing operations. These visits are not yet structured. We have asked the visiting specialists to include teaching similar and standardized surgical topics, not only to the COs but also the medical staff (continuing medical education for everyone).

Professor Nyengo Mkandawire, head of the Surgical Department of the College of Medicine and Member of the Malawi Medical Council, has advised the 4 Central Hospitals to make use of the Powerpoint presentations, both as teaching aids and as a means of ensuring that all district hospitals learn similar protocols. Meanwhile we are fully supporting an initiative taken by clinical officers to organize themselves by the establishment of a Clinical Officers Association.

\section{Review}

A Mid-term Assessment (2006) of the CO training was performed by Prof Cameron Bowie of the Department of Community Health of the College of Medicine ${ }^{7}$. He wrote " This mid-term review of the Clinical Officer Surgical Training Programme finds overwhelming support for the course. At each hospital clinical officer surgical skills have improved and patient care has benefited. The programme provides excellent continuing professional development for clinical officers. It is recommended that the programme be extended to the Northern and Central regions of Malawn"

\section{Future}

On the basis of Prof Bowie's review, the training programme was extended to the Northern Region and a follow-up programme has been established for the clinical officers in the Southern region. (2007-2009). Most of the on-the-job training visits to District Hospitals in the Northern Region now last 2 days. A gynaecologist is now reinforcing the project, as many procedures to be done involve emergency obstetrics.

The Mzuzu University have embraced the training at university level and have expressed their wish to upgrade the training to a bachelor degree course ${ }^{8}$. The Ministry of Health has given the Mzuzu University the green light to present its future plans and to address the $\mathrm{MOH}$ with a curriculum for a diploma course. If the University is successful, this will bring the clinical officers in Malawi an important boost in their career prospects. We believe that this will be of great benefit to clinical services in Malawi in general and to the management of surgical and gynaecological patients in particular.

Dr Peter Jiskoot was the initiating surgeon and Project manager for the Clinical Officer Training programme in Malawi. Other rotating specialists involved in running the CO Project were Dr. Jan Petit, surgeon and Dr.Ynze Rijken, gynaecologist.

\section{Acknowledgements}

Many thanks to those who have supported the programme:

- The Department of Surgery and Orthopaedics of the QECH, Blantyre especially Prof Nyengo Mkandawire, Prof Eric Borgstein and Dr Evert van Hasselt.

- The College of Medicine especially the Division of Community Health (Prof Cameron Bowie). Staff members of the Department of Obstetrics and Gynaecology (Dr G Kafulafula) and the Department of Anaesthesiology (C.Goddia)

- The surgeons who have visited the hospitals in the Southern Region and who were involved in the attachment weeks: Dr Marcel Schutgens, Dr Jim Wilde, Dr Charles Boissevain, Dr Henning Mothes and Dr Rita Peller 
- The Christian Health Association of Malawi: Francis Gondwe and Desiree Mhango

- The University of Mzuzu: Prof Peter Mwanza, Prof Yohane Nyasulu and Bill Mvalo

- The Ministry of Health in Lilongwe and the Zone Health Inspectors, Dr Esther Ratsma and Dr Gonani

- The Malawi Colleges of Health Sciences

- NGO's in the Netherlands: ICCO (Interchurch Organisation for Development Co-operation), Simavi, the Harrie van de Brekel Foundation, institutions, service clubs, churches, and many individuals for their financial support

- Illovo Sugar Company and Siku Transport in Malawi for hosting us free of charge

- EMC, leren in bedrijf, The Netherlands, for modifying our Powerpoint presentations

\section{Editor's Top 10 Picks}

1. Morbidity and mortality among a cohort of human immunodeficiency virus type 1 -infected and uninfected pregnant women and their infants from Malawi, Zambia, and Tanzania

Chilongozi D, Wang L, Brown L et al.

Pediatr Infect Dis J. 2008;27(9):808-14.

The study obtained data from mothers and their infants enrolled in a trial of antibiotics to reduce mother-to-childtransmission of HIV-1. The study found a high mortality among infants with early HIV-1 infection, this the authors say supports access to HIV-1 diagnostics and appropriate early treatment for all infants of HIV-1-infected mothers. The authors conclude a significant association between stage of maternal HIV-1 infection and infant mortality supports routine CD4 counts at the time of prenatal HIV-1 testing.

\section{Long term outcome of severe anaemia in Malawian children}

Phiri KS, Calis JC, Faragher B et al.

PLoS ONE. 2008;3(8):e2903.

The study assessed the short and long term outcome of severe anaemia in Malawian children and identified potential risk factors for death and further severe anaemia. Children were followed up for 18 months to compare all cause mortality and severe anaemia recurrence rates and to identify risk factors for these adverse outcomes. The authors concluded that severe anaemia carries a high 'hidden' morbidity and mortality occurring in the months after initial diagnosis and treatment. Because severe anaemia is very common, this is likely to contribute importantly to overall under-five mortality.

\section{Orthopaedic clinical officer program in} Malawi: a model for providing orthopaedic care

Mkandawire N, Ngulube C, Lavy C.

Clin Orthop Relat Res. 2008;466(10):2385-91.

Specialized orthopaedic clinical officers have been trained since 1985 and are deployed primarily in rural district hospitals to manage $80 \%$ to $90 \%$ of the orthopaedic workload in Malawi. They are trained in conservative management of most common traumatic and nontraumatic

\section{References}

1. Hornby P, Ozcan S (2003). Human resources for health sector strategic plans for Malawi, 2003 to 2013. Ministry of Health, Malawi.

2. World Health Organization (2005) The World Health Report 2005. Geneva

3. National Statistical Office. Malawi demographic health survey 2004

4. Steinlechner C, Tindall A, Lavy C, Mkandawire N, Chimangeni S., Surgery at the district hospital - what is going on? A national survey of surgical activity in the district hospitals in Malawi. Malawi Medical Journal 2004

5. Jiskoot P. On-the-job training in surgical and gynaecological procedures for Clinical Officers in Malawi. Project description

6. Phiri NA (2007). Does training in surgical skills of Clinical officers in the Southern region of Malawi reduce number of surgical referrals from District government/CHAM hospitals to Central Hospitals? MPH dissertation.for College of Medicine, University of Malawi

7. Bowie C (2006). Mid-term review of the clinical officer surgical training programme, Southern Malawi.

8. Vice Chancellor's meeting with the Minister of Health, December 2007

musculoskeletal conditions. Since the program began, 117 orthopaedic clinical officers have been trained, of whom 82 are in clinical practice. Orthopaedic clinical officer training is a cost-effective way of providing trained healthcare workers to meet the orthopaedic needs of a country with very few doctors and even fewer orthopaedic surgeons.

\section{World Health Organization Clinical Stage 3 disease conditions in HIV-infected patients who start antiretroviral therapy in Malawi}

Ngoma D, Makombe SD, Kamoto K, Harries AD.

Trop Doct. 2008;38(3):159-60.

A study was conducted to obtain information about disease conditions that are diagnosed in patients diagnosed as having World Health Organization Clinical Stage 3 HIV who are started on antiretroviral therapy (ART) in Africa. Several conditions were found amongst patients: unexplained weight loss, chronic fever or chronic diarrhoea, TB and bacterial infection. No patient was diagnosed as having haematological abnormalities. Nearly half the patients started on ART had a symptomatic, unspecified disease, (which may be obscuring important pathologies such as TB) and almost no laboratory assessment had taken place before the commencement of ART. These two areas need to be addressed in order to improve the management of patients starting on ART.

\section{HIV-positive women's experiences of a PMTCT programme in rural Malawi}

Kasenga F, Hurtig AK, Emmelin M

Midwifery. June 2008

The authors explored women's experiences of a prevention of mother-to-child transmission (PMTCT) programme in rural Malawi. An exploratory, qualitative study was conducted using in-depth interviews with 24 purposively selected women infected with human immunodeficiency virus. The authors conclude the PMTCT programme influences women's lives profoundly, and the importance of quality counselling and strengthening male involvement needs to be stressed as the programme is implemented by an increasing number of service providers.

continued on page 85 